\title{
Resolución de problemas y regulación del aprendizaje
}

\section{Problem solving and regulation in learning}

\author{
Jordi Deulofeu Piquet ${ }^{1}$ \\ Jordi.Deulofeu@uab.cat \\ JoAna Vilallonga Pons \\ jvillalongapo@uoc.edu \\ Universitat Autònoma de Barcelona, España
}

\section{Resumen:}

El desarrollo de la resolución de problemas en el aula presenta múltiples facetas, una de las cuales se refiere a la evaluación y particularmente al uso de instrumentos evaluativos que contribuyan a la mejora del aprendizaje y que sirvan al propio resolutor para reflexionar sobre sus producciones y su manera de proceder. En este artículo se muestra como el uso de una base de orientación para la resolución de problemas puede constituir efectivamente una ayuda cuando los alumnos resuelven problemas. En particular se analiza la relación entre el número de acciones de la base de orientación que cada uno de los alumnos ha tomado en consideración al resolver un problema y sus soluciones al problema. La existencia de dicha relación permite considerar la base de orientación como un andamiaje educativo para la resolución de problemas.

\section{Palabras clave:}

Resolución de problemas; evaluación reguladora; regulación del aprendizaje; base de orientación.

\begin{abstract}
:
Problem solving classroom practices have multiple facets. One of them is related to the evaluation and, consequently, the use of evaluation devices that enhance students' learning as they help the students to reflect on their own outcomes. In this article we analyse the use of an orientation basis as a device to support first year secondary students' mathematical problem solving. In particular, we study the relation between the number of actions of the orientation basis in which the students were involved when they solved a problem and their solutions to the problem. The emerging existence of this relationship allows us to consider the orientation basis as a problem-solving-related form of selfscaffolding.
\end{abstract}

\section{Keywords:}

Problem solving; competence assessment; regulation in learning; orientation basis.

\section{Dirección para correspondencia (correspondence address):}

Jordi Deulofeu Piquet. Departamento Didáctica de la Matemática i de las Ciencias. Facultad de Educación. Edificio G5, despacho 132. Universidad Autónoma de Barcelona. Cerdanyola del Vallès, 08193 


\section{Résumé:}

Le développement de la résolution de problèmes dans la salle de cours présente multiples facettes, une desquelles se rapporte à l'évaluation et particulièrement à l'usage d'instruments d'évaluation qui contribuent à l'amélioration de l'apprentissage et qui servent au propre résolveur pour réfléchir sur leurs productions (usage de l'instrument d'évaluation comme feedback et je crois que ceci est fondamental). Dans cet article on démontre comment l'usage d'une base d'orientation pour la résolution de problèmes peut constituer effectivement une aide quand les élèves résolvent des problèmes. En particulier on analyse la relation entre le nombre d'actions de la base d'orientation que chacun des élèves a pris en considération à l'heure de résoudre un problème et de donner ses solutions au problème. L'existence de dite relation permet de considérer un échafaudage éducatif pour la résolution de problèmes.

\section{Mots clés:}

Résolution de problèmes; évaluation des compétences; autorégulation de l'apprentissage; bases de l'orientation.

Fecha de recepción: 9-5-2018

Fecha de aceptación: 25-10-2018

\section{Introducción y objetivo}

La resolución de problemas es un aspecto fundamental, tanto de las matemáticas como de la educación matemática. Como menciona Halmos (1980), los problemas son el corazón de las matemáticas y, de manera paralela, lo afirma de ellos el NCTM (1980) al referirse a la enseñanza de las matemáticas. Con ello se manifiesta un doble nivel de complejidad de la resolución de problemas, ya sea entendida como una actividad por sí misma, como un modo de enseñar matemáticas (Mason, 2016a).

Sin embargo, ha trascurrido mucho tiempo desde la década de los ochenta y ha habido períodos en los que dicha importancia se ha reflejado en la investigación en educación matemática, mientras que en otros no (Puig, 2008).

En el siglo XXI, con las múltiples reformas de los currículos, con enfoques centrados en procesos (NCTM, 2003) o bien en competencias (Niss y Højgaard, 2011), la resolución de problemas ha vuelto a emerger con fuerza convirtiéndose en un objetivo importante de la investigación educativa actual, en la que destacan proyectos de investigación de diseño a largo plazo que trabajan con grupos de docentes en activo para establecer pautas prácticas para iniciativas sostenibles de resolución de problemas (Stacey, 2016).Posiblemente esto ha sido así porque tanto al 
estructurar los grandes procesos matemáticos, como al determinar cuáles son las subcompetencias que conforman la competencia matemática, la resolución de problemas ha aparecido siempre y de manera inevitable como uno de las principales en la enseñanza de las matemáticas y, en cualquier caso, es un caracterizador del quehacer matemático (Carrillo y Guevara, 1996).

De acuerdo con las reflexiones de Mason (2016b), entendemos que algo o alguna situación es un problema cuando quien pretende resolverlo experimenta un estado de dificultad, asume la tarea de dar sentido a la situación y se involucra en alguna actividad creativa con sentido para afrontarlo. La dificultad principal radica en aquello que hace que una situación sea problemática para los estudiantes, así como en entrever lo que va a movilizar la actividad al tener en mente posibles acciones. Vinculado a ello, se puede decir mucho aún sobre las prácticas en el aula, en particular en relación a cuándo intervenir y de qué manera hacerlo en las tareas que se pueden proponer a los estudiantes (Mason, 2016b).

Al desarrollar la resolución de problemas matemáticos se ponen de manifiesto y toman significado prácticamente todos los aspectos trabajados en la educación matemática, tanto los contenidos conceptuales, como las técnicas, sin olvidar los propios contenidos de la resolución de problemas, principalmente las heurísticas. Por ello se considera la resolución de problemas como el eje central de cualquier proceso de enseñanza y aprendizaje de las matemáticas (Ponte, 2007; Schoenfeld, 1992).

A lo largo de estos últimos 30 años ha habido avances significativos en la comprensión de los aspectos cognitivos, metacognitivos y afectivos de la resolución de problemas en matemáticas y también ha habido una considerable investigación sobre la enseñanza de la resolución de problemas matemáticos en las aulas. Sin embargo, quedan aún más preguntas que respuestas sobre esta compleja actividad (Lester y Cai, 2016). De hecho, al analizar la gestión del aula cuando la actividad principal es la resolución de problemas encontramos numerosos aspectos en los que es necesario centrarse para tratar de mejorar dicha gestión. Preguntas del tipo, ¿cómo evitar las situaciones de bloqueo?, ¿cómo lograr que los alumnos identifiquen sus errores y traten de corregirlos?, ¿cómo lograr una revisión del proceso de resolución que lleve a una argumentación sobre la validez del mismo?, ¿cómo conseguir que los alumnos sean conscientes de las acciones que llevan a cabo para resolver un problema y las vayan integrando de manera consciente y coherente?, ¿hay relación 
entre esas acciones y sus resoluciones?, son algunas de las que nos preocupan y para las que todavía tenemos respuestas muy incipientes.

Por otra parte, si entendemos la evaluación como una parte sustancial del proceso de aprendizaje, centrada en la obtención de datos para poder analizar y reconducir las dificultades que se detectan, tanto sobre aqueIlo que hay que hacer para resolver una determinada tarea de resolución de problemas como aquello que uno ha hecho al tratar de resolverla, resulta esencial disponer de instrumentos adecuados que posibiliten la consecución de dichos objetivos. Ante las carencias reportadas sobre la forma tradicional de evaluación, en cuanto que ésta no puede revelar realmente lo que los estudiantes aprenden y conocen, se desprende la necesidad de cambios en la evaluación que permitan una evaluación reguladora (Sanmartí, 2010) de las competencias más complejas vinculadas a la resolución de problemas (Rosli, Goldsby y Capraro, 2013).

El interés por caracterizar la competencia en resolución de problemas en un marco competencial de la educación, y ante el papel relevante que adquiere la evaluación en este marco educativo, conduce a cuestionarnos por la generación y aplicación de instrumentos para la resolución de problemas en las aulas con la finalidad que desarrollen en los alumnos la regulación de su propio aprendizaje. De acuerdo con ello, nos preguntamos cómo se puede elaborar un instrumento con finalidad reguladora para ayudar a desarrollar la competencia de resolución de problemas matemáticos de los alumnos, y qué efectos, y en qué medida, puede tener su aplicación en el desempeño de los alumnos al resolver un problema.

El presente artículo pretende mostrar como el uso una base de orientación para la resolución de problemas, entendido como un instrumento para una evaluación formadora y elaborada mediante un proceso de refinamientos sucesivos a partir de las propias aportaciones de los alumnos, puede constituir una ayuda en el sentido deseado cuando los alumnos resuelven problemas. En particular, se analiza la relación entre el número de acciones de la base de orientación que cada uno de los alumnos ha tomado en consideración y el éxito de su resolución. La existencia de dicha relación permite considerar la base de orientación, que tiene las características de lo que se conoce como un andamiaje educativo, como una herramienta útil para aprender a resolver problemas. 


\section{Marco conceptual}

Desde finales de los años 70, la comunidad investigadora ha trabajado de manera destacada para caracterizar la resolución de problemas, al mismo tiempo que estudiar la gestión de los procesos de enseñanza y aprendizaje que permitan a los estudiantes convertirse en mejores resolutores (Ponte, 2007). Sin embargo, la noción de problema matemático ha sido utilizada de múltiples maneras, desde la realización de ejercicios rutinarios hasta el desarrollo de la actividad matemática profesional (Schoenfeld, 1992). Esta variedad de tareas, junto a la dificultad en ocasiones para determinar si estamos ante la resolución de un problema, ha llevado a los educadores de matemáticas a sentirse incómodos con respecto a este concepto (Ponte, 2007).

El gran volumen de referencias sobre la resolución de problemas matemáticos pone de manifiesto que la resolución de problemas es una actividad compleja de llevar a cabo en el aula, tanto para estudiantes de cualquier edad (Mason, Burton y Stacey, 1982; Pólya, 1945) como para docentes que intentan crear ambientes de trabajo adecuados (De Corte y Verschaffel, 2003; Schoenfeld, 2013). Se apunta que parte de esta dificultad radica en la habilidad de los estudiantes, como aprendices, para transferir el proceso de resolución de un problema a otras situaciones (Coltman, Petyaeva y Anghileri, 2002). Sin embargo, las evidencias muestran que una intervención adecuada puede ayudar a tomar conciencia de la importancia, no sólo de hallar la solución de un problema, sino de los procesos que permiten llegar a ella (Coltman et al., 2002; Sanmartí, 2007) algo fundamental en la resolución de problemas si tenemos en cuenta el papel predominante que parece tomar la autorregulación para la resolución de problemas matemáticos de manera exitosa (Panaoura, 2016).

Ante una perspectiva socioconstructivista de la educación, sobre la que está constituida el currículum catalán actual (Departament d'Ensenyament, 2016), y en la que se considera que el conocimiento se construye mediante un proceso en el que las ideas pueden evolucionar gracias a situaciones problemáticas que favorecen la explicitación y el contraste de puntos de vista, la evaluación es un elemento fundamental de la dinámica del proceso de aprendizaje (Sanmartí y Jorba, 1995). Para que esta intervención sea adecuada y significativa en el proceso de enseñanza y aprendizaje, se requiere de un proceso de evaluación 
que permita analizar el estado del estudiante de acuerdo con las tareas propuestas. Se desprende, con ello, la necesidad de una evaluación que preste atención a la observación y el cuestionamiento de los alumnos, tanto para evaluar su comprensión como para tener en cuenta sus sentimientos y sus creencias acerca de lo que están trabajando (Lester y Kroll, 1991). Bajo una concepción competencial de la educación, apreciamos que la capacidad de análisis sobre los procesos de resolución junto con una aplicación adecuada de ellos es, en gran parte, la esencia de una educación en la que se pretende que los alumnos adquieran la competencia en resolución de problemas y puedan seguir desarrollándola, de manera autónoma, en ocasiones posteriores.

De acuerdo con ello, observamos que para lograr que los alumnos alcancen los objetivos mencionados se requiere de un proceso evaluativo doble. En efecto, de acuerdo con la doble complejidad que la resolución de problemas conlleva (Mason, 2016a), por un lado es necesaria una evaluación de los procesos del propio estudiante, como resolutor, y por otro lado, al tratarse de un objetivo educativo, es fundamental una evaluación, en tanto que aprendiz, que vaya garantizándole la adquisición de la misma.

Sin embargo, como ya se ha comentado, la forma tradicional de evaluar la resolución de problemas, basada principalmente en una evaluación final y, especialmente, calificadora del desempeño de los alumnos por parte de los docentes, no puede realmente revelar lo que los estudiantes aprenden (Rosli et al., 2013; Sanmartí, 2010). En respuesta a ello, surge, de forma natural, la necesidad de una evaluación reguladora (Lester y Kroll, 1991; Sanmartí, 2010) de la resolución de problemas y, por consiguiente, el establecimiento de instrumentos y de su implementación que permitan desarrollarla de manera consecuente.

En este sentido, se distinguen dos finalidades de la evaluación. Por un lado, una función identificada como calificadora, cuyo objetivo recae principalmente en el establecimiento de grados de aprendizaje. Por otro, la que pretende actuar como medio para regular los aprendizajes y de ahí que se hable de su función reguladora. A su vez, esta función reguladora puede ser canalizada de dos modos: de manera formativa, cuando las decisiones son tomadas fundamentalmente por los docentes, o en modo formadora, cuando las decisiones son tomadas principalmente por el estudiante (Sanmartí, 2010) y; por tanto, vinculadas a procesos de retroalimentación y autorregulación. 
Para alcanzar de manera satisfactoria las finalidades de la evaluación, cabe disponer de información de distinta naturaleza y para ello es interesante disponer de distintas estrategias e instrumentos evaluativos (Lester y Kroll, 1991; Sanmartí, 2010). Para una evaluación de la educación matemática, Lester y Kroll (1991) destacan la utilidad de recoger información a través de cuatro estrategias: (1) observar y cuestionar a los alumnos, (2) valorar el trabajo (de matemáticas) de los alumnos, (3) usar la escritura de los alumnos para la evaluación, y (4) valorar el trabajo de los alumnos a través de portafolios individuales. Por su parte, Danielson (1997) apuntó al uso de rúbricas para la evaluación de actividades matemáticas en distintas etapas de la educación obligatoria por su pertinencia para examinar la habilidad de los alumnos para resolver problemas.

Las rúbricas se han caracterizado como una herramienta de gran potencialidad didáctica, capaz de contribuir significativamente en la mejora de un proceso: evaluación-enseñanza-aprendizaje (Blanco, 2008; Rosli et al., 2013; Sanmartí, 2010), por la versatilidad que presentan como instrumento de evaluación y por su capacidad de ajustarse a las exigencias de una evaluación de competencias multidimensional y polifacética (Blanco, 2008) que ayudan a los estudiantes a adquirir competencias complejas (Rosli et al., 2013; Smit, Bachmann, Blum, Birri y Hess, 2017; Sanmartí, 2010), como puede ser la resolución de problemas.

Así, centradas en los objetivos de aprendizaje, y por tanto, instrumentos de carácter formativo (Sanmartí, 2010), las rúbricas se describen como un conjunto coherente de criterios para el trabajo de los estudiantes que incluye descripciones de niveles de la calidad del desempeño. Así, en base a la información de la rúbrica, y mediante interpretaciones basadas en criterios, los maestros pueden monitorear el aprendizaje de los estudiantes y proporcionar algunas retroalimentaciones para que los estudiantes puedan reestructurar sus conocimientos (p.ej. Rosli et al., 2013; Smit et al., 2017; Sanmartí, 2010).Por su parte, las bases de orientación se definen como aquellos instrumentos que resumen de manera gráfica y ordenada las acciones que se pretende realizar, con la finalidad de promover que los estudiantes anticipen y planifiquen aquello que han de llevar a cabo para resolver con éxito distintos tipos de tareas escolares (Sanmartí, 2007).

Por otro lado, tenemos las bases de orientación que se centran en las acciones a realizar, necesarias para desempeñar una tarea, ejemplificando lo que el proceso de enseñanza debería transmitir, y por ello se en- 
tienden como instrumentos evaluativos de carácter regulador y formador (Sanmartí, 2010). Cada persona tiene una forma personal de aprender que construye progresivamente a lo largo de los años; sin embargo, (Jorba y Sanmartí, 2004). Sin embargo, es posible ayudar a los alumnos en la construcción de este sistema personal (Jorba y Sanmartí, 2004), para lo cual es necesario incidir en la aturorregulación por parte de los propios alumnos, entendida como la representación de las propias capacidades y formas de aprender. En este sentido, ya se han encontrado usos positivos de bases de orientación en competencias lingüísticas complejas (Sanmartí, 2010), pero todavía no en educación matemática.

A pesar de la aparente positividad del uso de estos instrumentos, las referencias en relación con su implementación, en particular, para la resolución de problemas en la enseñanza obligatoria, así como análisis relativos a su eficacia, son aún escasas, especialmente en el caso del uso de las bases de orientación.

Paralelamente, al indagar entre los instrumentos que pudieran aportar una mejora en la gestión de la adquisición de la competencia en resolución de problemas, encontramos referencias a la metáfora del scaffolding (Wood, Bruner y Ross, 1976), que traducimos al español como andamiaje educativo. Tomado del campo de la construcción, el andamiaje educativo se define como aquel apoyo (temporal) que permite al que aprende a volverse progresivamente independiente, objetivo que se identifica con el que persigue una educación por competencias.

Ante ello nos interesamos en qué sentido una base de orientación, construida de acuerdo con los elementos esenciales de procesos de autorregulación (Jorba y Sanmartí, 1995), puede conllevar un andamiaje efectivo de la resolución de problemas. Más aún, aunque conscientes de su dificultad (van de Pol, Volman, y Beishuizen, 2010), indagamos en cómo evaluar la eficacia de una base de orientación concreta construida como andamiaje educativo para la resolución de problemas matemáticos.

\section{Metodología de la investigación}

El estudio que se presenta deriva de una investigación más amplia sobre el diseño y uso de las bases de orientación como instrumentos autorreguladores del aprendizaje para la resolución de problemas en matemáticas (Villalonga Pons, 2017). 
De acuerdo con un proceso de investigación-acción que comprendió tres ciclos, se trabajó para la elaboración e implementación de una base de orientación para la resolución de problemas para alumnos de $6^{\circ}$ de Educación Primaria y $1^{\circ}$ de Educación Secundaria, así como el análisis de uso (Villalonga Pons, 2017). Si bien en el primer ciclo se trabajó, fundamentalmente, para obtener evidencias del uso de una base de orientación para la resolución de problemas en un marco cualitativo (Villalonga Pons y VIIlalonga Pons y Deulofeu Piquet, 2015), en el segundo se analizó la necesidad de las dimensiones y acciones que un instrumento como tal debería comprender (VIllalonga Pons y Deulofeu Piquet, 2017a y 2017b). Finalmente, en el tercer ciclo de implementación, se trató de evidenciar hasta qué punto era posible establecer relaciones entre el uso de la base de orientación por parte de los alumnos y el éxito de sus resoluciones (VIllalonga Pons y Andrews, 2017 y 2018). De ello surgieron tres bases de orientación, cada una como un refinamiento sucesivo de la anterior y, de manera paralela el análisis del propio proceso de refinamiento.

El presente estudio surge de la tercera de estas implementaciones, en la que se utilizó la tercera de las bases de orientación diseñadas y utilizadas a lo largo del estudio. Se trata de la base de orientación que se detallada en la Tabla 1 y a la que, de ahora en adelante, nos referiremos como BO. Ésta fue diseñada atendiendo las características de andamiaje educativo, como un instrumento de retroalimentación contingente, que facilita y avanza sugerencias sobre las acciones a desarrollar, centrada en facilitar su disipación y la transferencia de responsabilidad (van de Pol et al., 2010).

En cuanto a su estructura, y bajo la influencia de Pólya (1945), la base de orientación presenta cuatro dimensiones (entiendo el problema, trazo un plan de acción, aplico mi plan de acción, reviso mi tarea), entendidos como niveles de implicación del resolutor en la resolución del problema. Vinculadas a ellos, se sugieren doce acciones que se espera que los alumnos tomen en consideración. Cada una de estas acciones, así como el modo de describirlas, deriva de las observaciones de los comportamientos de resolución de problemas de los alumnos realizadas en las implementaciones anteriores y de acuerdo con las estrategias de resolución de problemas encontradas en la literatura (p. ej., De Corte et al., 2000; Mason et al., 1982). Finalmente, aparece una tercera columna en la que se espera que los alumnos anoten las acciones que consideran haber tomado en consideración. 
Tabla 1. Base de orientación (BO) traducida al español del original catalán.

\begin{tabular}{|c|c|c|}
\hline DIMENSIONES & ACCIONES & SEGUIMIENTO \\
\hline \multirow{3}{*}{$\begin{array}{l}\text { Entiendo el } \\
\text { problema }\end{array}$} & A1. He leído lo que se expone, al menos dos veces. & \\
\hline & A2. Entiendo lo que pretende el problema & \\
\hline & A3. He identificado y he entendido los datos & \\
\hline \multirow{3}{*}{$\begin{array}{l}\text { Trazo un plan de } \\
\text { acción }\end{array}$} & A4. He jugado con los datos & \\
\hline & A5. He preparado una estrategia de resolución. & \\
\hline & $\begin{array}{l}\text { A6. He comprobado que mi estrategia encaja bien con } \\
\text { los datos. }\end{array}$ & \\
\hline \multirow{3}{*}{$\begin{array}{l}\text { Aplico mi plan de } \\
\text { acción }\end{array}$} & A7. He implementado miestrategia. & \\
\hline & $\begin{array}{l}\text { A8. He recopilado todas mis acciones de manera que las } \\
\text { entiendo. }\end{array}$ & \\
\hline & $\begin{array}{l}\text { A9. He recopilado todas mis acciones de modo que los } \\
\text { otros las entiendan. }\end{array}$ & \\
\hline \multirow{3}{*}{ Reviso mi tarea } & A10. Cuando me atasco vuelvo al principio & \\
\hline & $\begin{array}{l}\text { A11. Cuando he finalizado, he comprobado mis } \\
\text { respuestas. }\end{array}$ & \\
\hline & $\begin{array}{l}\text { A12. He explorado otras respuestas y/o mejores } \\
\text { soluciones. }\end{array}$ & \\
\hline
\end{tabular}

El caso que se presenta corresponde al uso, y un análisis concreto de su uso, de la BO en cuestión, en un grupo de alumnos de 28 estudiantes de $1^{\circ}$ de Educación Secundaria Obligatoria de un centro educativo de Cataluña. El docente involucrado es físico de formación y cuenta con 15 años de experiencia como profesor de matemáticas en Educación Secundaria. De sus clases habituales destaca esencialmente el trabajo con los problemas que aparecen en el libro de texto, para los que, hasta el momento, no disponía de instrumentos que le permitieran una evaluación reguladora de la resolución de problemas.

La práctica se desarrolló en el tercer trimestre del curso académico 2015-2016. De acuerdo con la programación del docente, y aproximadamente con una distancia de un mes entre ellos, se plantearon a los estudiantes tres problemas desconocidos y no rutinarios (ver Anexo) junto con instrucciones para el uso de la BO. El docente eligió el orden para trabajarlos. En las otras lecciones de este período, los alumnos no trabajaron otros problemas que los de su libro de texto ni consideraron la base de orientación para ninguna de sus prácticas habituales.

Cada problema fue propuesto en una sesión de clase distinta, invitando a los alumnos a utilizar la BO para resolver el problema en cuestión. En cada sesión, los alumnos recibieron una copia en papel del problema en el que tenían que escribir su propia resolución así como una copia 
de la BO para guiar su actividad al mismo tiempo que pudieran registrar las acciones que consideraban abordar.

Para garantizar, en la medida de lo posible, que los alumnos entendían el vocabulario, estructura y propósito general de la BO, previamente a la primera vez que se utilizó, el docente explicó a los alumnos el propósito de la $\mathrm{BO}$ y, conjuntamente con la clase, discutieron y aclararon el significado y el propósito de cada elemento de la BO.

En este artículo se presentan los datos obtenidos al resolver el segundo (ver Figura 2 del Anexo) de los problemas propuestos, haciendo uso de la BO. La elección se debe al hecho que no es la primera vez que usan la $\mathrm{BO}$ (tanto el docente como los alumnos ya habían tenido un primer contacto con la BO) y tampoco es la culminación de todo el proceso, con el objetivo de estudiar la aplicación de las acciones que comprende la $\mathrm{BO}$ en la práctica de los alumnos, de acuerdo con sus anotaciones.

Los datos analizados corresponden a las anotaciones tomadas por los propios alumnos, tanto en la hoja donde tenían impresa la BO y donde se pedía que registraran, por sí mismos y de acuerdo con su práctica, las acciones que creían ir aplicando, y las resoluciones que incluyen las respuestas a los problemas, que debían realizar por escrito en la hoja donde se había facilitado el problema impreso.

Constatadas evidencias cualitativas sobre el uso de una base de orientación para la resolución de problemas (Villalonga Pons y Deulofeu Piquet, 2017a y 2017b), y atendiendo a la información que se desprende de la $3^{\mathrm{a}}$ columna de la BO, para el análisis se consideró la aplicación de una herramienta estadística con la intención de establecer posibles vínculos entre las acciones realizadas por los alumnos y sus respuestas a los problemas. Atendiendo el tamaño de la muestra y de acuerdo con los estudios anteriores (Villalonga Pons y Andrews, 2017 y 2018), se optó por aplicar el test exacto de Fisher, al ser una prueba de significación estadística para muestras pequeñas, especialmente útil cuando no tiene sentido aplicar el test de la Chi Cuadrado.

El Test exacto de Fisher pretende examinar el grado de asociación probable (o contingente) entre dos grupos de datos categóricos. Se trata de un contraste de hipótesis cuyo objetivo es probar si los dos grupos que se consideran son o no independientes entre sí. Se utiliza, fundamentalmente, en la comparación de dos grupos respecto a una variable dicotómica o en la valoración de la relación entre dos variables cualita- 
tivas, ambas dicotómicas. Así mismo, la prueba es extensible a variables que presentan más de dos propiedades o cualidades. Otra de las particularidades de esta prueba es la posibilidad de calcular el valor de la desviación de la hipótesis nula $\left(\mathrm{H}_{0}\right)$ de manera exacta.

Esta prueba se basa en la distribución hipergeométrica (Llopis, 2013), caracterizada por seguir una situación en la que hay $N$ posibles observaciones, distribuidas en dos tipos distintos en proporción $r$ y $N-r$, al realizar $k$ observaciones sin repetición, pretende ver cuántas de estas $k$ observaciones serán de un tipo o de otro. Para aplicar el Test exacto de Fisher, conviene, entonces, distribuir las propiedades de las dos variables en una tabla de contingencia, con lo que, el estudio se puede percibir también como un problema de comparación de proporciones. En este sentido, el Test exacto de Fisher busca, a partir de los datos, qué combinaciones serían más extremas que la observada, entendiendo por extremas aquellas que presentan mayor diferencia entre los porcentajes o mayor asociación entre los valores de las dos variables de las que se pretende estudiar su relación. Así, si es el valor de la probabilidad que determina la independencia, se concluye:

- $p<0,05$ : se rechaza la hipótesis nula $\mathrm{H}_{0}$ y se asume que las dos variables están relacionadas

- $p>0,05$ : los datos son consistentes con la hipótesis nula $\mathrm{H}_{0}, \mathrm{y}$ no hay evidencia de dependencia

Para el estudio se utilizó el software de acceso público desarrollado por Kirkman (1996).

\section{Resultados y discusión}

En la Tabla 2 se muestran los datos correspondientes a los 28 alumnos del grupo de $1^{\circ}$ de ESO participantes al utilizar la BO mientras trabajaban en el problema de la Figura 1. La columna de la izquierda, con las referencias $\mathrm{A} 1, \ldots, \mathrm{A} 12$ alude a las 12 acciones que comprende la BO utilizada. Los puntos $(\bullet)$ corresponden a las marcas de seguimiento escritas por cada uno de los alumnos en el registro de la BO. Las casillas correspondientes a la fila \#A recogen el total de las acciones de la $\mathrm{BO}$ que cada alumno marcó para indicar que se había involucrado en ella. El problema utilizado presenta dos cuestiones. Por esto las casillas de la 
fila \#C contienen un 2 cuando el alumno obtuvo soluciones correctas a ambas preguntas, un 1 si sólo obtuvo una solución correcta a una de las preguntas y un 0 cuando no obtuvo ninguna solución correcta para ninguna de las cuestiones. Finalmente, las casillas de la última fila contienen una $\mathrm{F}$ cuando las evidencias de la resolución muestran que el alumno dio por finalizado el problema, o $\mathrm{N}$ en caso contrario.

Tabla 2. Seguimiento de la $B O$ al resolver el problema de la Figura 1 y sus soluciones.

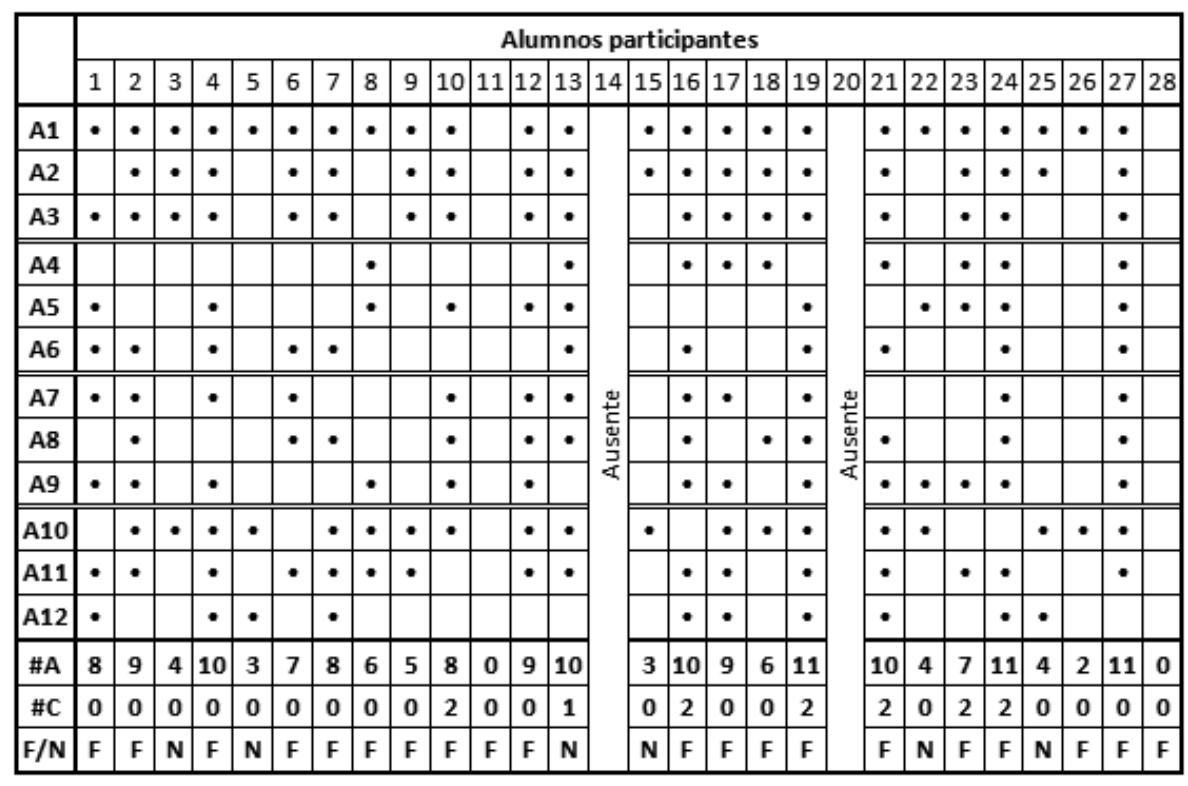

Los datos de la Tabla 2 revelan que dos alumnos (11 y 28) no se involucraron con el uso la $\mathrm{BO}$, puesto que no marcaron ninguna de sus acciones, mientras que el resto de alumnos hicieron algún seguimiento de la $\mathrm{BO}$, en grados variables. Seis alumnos obtuvieron soluciones correctas en ambas partes del problema, un alumno obtuvo una solución correcta a una parte del problema y los restantes, incluyendo los que no completaron ninguna acción de la BO, no alcanzaron ninguna solución correcta a ninguna de las dos preguntas del problema.

Observamos que ningún alumno marcó las 12 acciones que comprende. Sin embargo, dos de los tres alumnos (19 y 24) que marcaron 11 de las 12 acciones obtuvieron soluciones correctas a las dos preguntas del problema. De hecho, los datos de la Tabla 2 muestran que cuando 
Ios alumnos $(10,16,19,21,23$ y 24$)$ dieron respuesta correcta a las dos partes del problema, éstos siempre completaron siete o más acciones de la BO. Interesados por el control de la propia actividad, observamos como de las acciones vinculadas a la comprobación de la BO, "A6. He comprobado que mi estrategia encaja bien con los datos" y "A11. Cuando he finalizado, he comprobado mis respuestas", once alumnos marcaron la A6, y dieciséis, que incluyen los once anteriores, marcaron la A11.

La Tabla 3 compara, para el total de alumnos, el número de acciones de la $\mathrm{BO}$ que los alumnos marcaron como aplicadas, con el número soluciones correctas.

Tabla 3. Relación entre el número de acciones de la BO y respuestas correctas.

\begin{tabular}{|r|c|ccc|}
\hline \multicolumn{2}{|c|}{28 intentos } & \#C: Número soluciones correctas \\
\cline { 3 - 5 } & 0 & 1 & 2 \\
\hline \#A: Número de acciones de la & $0-6$ & $\mathbf{1 1}$ & $\mathbf{0}$ & $\mathbf{0}$ \\
BO en qué se involucraron & $7-12$ & $\mathbf{8}$ & $\mathbf{1}$ & $\mathbf{6}$ \\
\hline
\end{tabular}

Test Exacto de Fisher $p=0.021$

Al aplicar el Test Exacto de Fisher en estos datos se obtiene un pvalor de $p=0.021$, lo que indica que tales cifras no sólo son improbables debido al azar, sino que los alumnos que no se implicaron en la BO tendieron a no completar el problema. Más concretamente, los valores de la Tabla 3 muestran claramente que los alumnos que completaron satisfactoriamente las dos cuestiones del problema siempre se habían involucrado con 7 o más de las 12 acciones que sugiere la BO.

Por su parte, en la Tabla 4 se comparan el compromiso de los alumnos con las acciones de comprobación, A6 y la A11 y el hecho de completar sus intentos de resolución.

Tabla 4. Relación entre el número de acciones de la $B O$ e intentos finalizados.

\begin{tabular}{|c|c|c|c|}
\hline \multirow{2}{*}{\multicolumn{2}{|c|}{28 intentos }} & \multicolumn{2}{|c|}{$\begin{array}{c}\text { Intentos Finalizados o No } \\
\text { Finalizados }\end{array}$} \\
\hline & & NF & $\mathrm{F}$ \\
\hline \multirow{2}{*}{$\begin{array}{r}\text { Compromiso con las acciones de } \\
\text { comprobación (A6/A11) }\end{array}$} & - & 5 & 5 \\
\hline & A6/A11 & 1 & 15 \\
\hline \multicolumn{2}{|c|}{ Test Exacto de Fisher } & & \\
\hline
\end{tabular}

Los datos de la Tabla 4 descubren, además de la improbabilidad que sean debidas al azar, que los alumnos que no se comprometieron con 
alguna de las acciones de comprobación tuvieron cinco veces más probabilidades de no finalizar la resolución que aquellos que se comprometieron con alguna de las acciones de comprobación. Los datos también muestran que los alumnos que completaron alguna de las acciones de comprobación de la BO mayoritariamente finalizaron su intento de resolución. En resumen, los alumnos que se comprometieron con alguna de las acciones de comprobación de la $\mathrm{BO}$ muestran más probabilidades de finalizar un intento de resolución que los estudiantes que no lo hicieron.

Sin embargo, como se desprende de la Tabla 2, muchos de los intentos de resolución, 19, terminaron en fracaso, un resultado que pide de ser analizada con más profundidad, y de ello se encargan las Tablas 5 y Tabla 6.

Tabla 5. Relación entre el número de acciones de la BO y los intentos fallidos finalizados.

\begin{tabular}{|c|c|c|c|}
\hline \multirow{2}{*}{\multicolumn{2}{|c|}{19 intentos fallidos }} & \multicolumn{2}{|c|}{$\begin{array}{c}\text { Intentos No Finalizados (NF) o } \\
\text { Finalizados (F) }\end{array}$} \\
\hline & & NF & $\mathrm{F}$ \\
\hline \multirow{2}{*}{$\begin{array}{r}\text { \#A: Número de acciones de la } \\
\text { BO en qué se involucraron }\end{array}$} & $0-6$ & 5 & 6 \\
\hline & $7-12$ & $\mathbf{0}$ & 8 \\
\hline \multicolumn{2}{|c|}{ Test Exacto de Fisher } & \multicolumn{2}{|c|}{$p=0.045$} \\
\hline
\end{tabular}

Los datos de la Tabla 5 muestran, para los 19 intentos fallidos, una comparación entre las acciones de la $\mathrm{BO}$ en las que los alumnos se implicaron, con la finalización, aunque fallidos, de sus intentos de resolución. En este sentido, los datos muestran que los estudiantes que completaron siete o más acciones OB completaron siempre su intento de solución mientras que los que nunca finalizaron sus intentos de resolución marcaron 6 o menos acciones de la BO. En resumen, incluso cuando sus soluciones fueron incorrectas, los alumnos que completaron siete o más acciones de la BO tuvieron muchas más probabilidades de completar un intento de resolución que los estudiantes que no lo hicieron.

También para los 19 intentos fallidos, los datos de la Tabla 6 muestran una comparación entre el total de acciones da la BO consideradas y el grado en que los alumnos se involucraron con las acciones de comprobación, la A6 y la A11. 
Tabla 6. Número de acciones de la BO y acciones de comprobación en los intentos fallidos.

\begin{tabular}{|r|c|c|c|c|}
\hline \multirow{2}{*}{19 intentos fallidos } & \multicolumn{3}{c|}{ Número de A6/A11 acciones } \\
\cline { 2 - 5 } \multicolumn{2}{|c|}{} & 0 & 1 & 2 \\
\hline \#A: Número de acciones de la BO & $0-6$ & $\mathbf{9}$ & $\mathbf{2}$ & $\mathbf{0}$ \\
\cline { 4 - 5 } en qué se involucraron & $7-12$ & $\mathbf{0}$ & $\mathbf{2}$ & $\mathbf{6}$ \\
\hline \multicolumn{2}{|r|}{ Test Exacto de Fisher } & \multicolumn{3}{|c}{$\mathbf{p}<\mathbf{0 . 0 0 1}$} \\
\hline
\end{tabular}

Los datos correspondientes al total muestran claramente, a un nivel de probabilidad que presenta el azar como una imposibilidad, que incluso cuando sus soluciones fueron incorrectas, los alumnos que completaron las acciones de comprobación (A6 y A11), completaron la mayoría de las acciones de la BO. Alternativamente, los estudiantes que no completaron estas dos acciones casi nunca completaron más de seis de las acciones de la $\mathrm{BO}$. En otras palabras, las dos acciones de comprobación de la BO, A6 y A11 parecen ser fuertes predictores del compromiso más amplio de un alumno con la $\mathrm{BO}$, incluso para aquellos estudiantes que fallan en la resolución del problema.

Analizar los datos a nivel de las cuatro fases en que se agrupan las acciones de la $\mathrm{BO}$, en lugar de como acciones individuales, ofrece también información interesante. En este sentido, los datos de la Tabla 2, muestran que a medida que se va avanzando en la $\mathrm{BO}$, disminuye el número de alumnos que completan cada dimensión. Como es de esperar, la mayoría de alumnos se involucraron con la primera dimensión, Entiendo el problema. En particular 24 alumnos iniciaron las tres acciones vinculadas a ella, aunque luego sólo 17 (70.8\%) de ellos llegaron a completar las tres. Con respecto a la segunda dimensión, 9 comenzaron con la primera acción, de los cuales 3 (33.3\%) completaron las tres. La tercera dimensión, "Aplico mi plan de acción", fue iniciada por 12 alumnos (tres más de los que completó la segunda), de los cuales 7 (58.3\%) la completaron. Por último, de los 19 alumnos que iniciaron la dimensión final, "Reviso mi tarea", sólo 4 marcaron haberla completado (21\%).

Los datos anteriores nos informan de hechos que consideramos relevantes en cuanto a la implicación de los alumnos en cada una de las dimensiones de la resolución establecidas en la BO. En primer lugar, los alumnos que comenzaron a trabajar en una dimensión no completaron siempre las tres acciones vinculadas a la misma. La primera dimensión, con la mayor tasa de implicación, parece menos problemática, en com- 
paración con el resto de ellas, especialmente la segunda y la cuarta, de las que cada una de ellas se desprende una acción de comprobación: la segunda, con el menor nivel de implicación y, la última, con la menor tasa de finalización. Con ello, observamos como un examen más preciso de las cuatro dimensiones puede también informar de futuros desarrollos de la BO.

\section{Reflexiones finales}

Con la experimentación desarrollada hemos tratado de hallar una manera de medir, más allá de los resultados cualitativos analizados con anterioridad, que nos proporcionaban indicios razonables sobre el efecto del uso de la base de orientación presentada en la Tabla 1 y su reconocimiento como auto-andamiaje de la resolución de problemas matemáticos.

Al proponer que los alumnos marcaran de manera explícita las acciones de la BO que aplicaban, en el papel donde la tenían impresa, ha permitido establecer una correspondencia directa entre las acciones marcadas y las soluciones al problema, correspondencia que ha posibilitado la introducción de un análisis cuantitativo a través del Test Exacto de Fisher.

Este estudio ha permitido precisar y ampliar las observaciones cualitativas realizadas con anterioridad, además de convertirse en una herramienta para valorar el instrumento en sí mismo. Con ello, destacamos el Test Exacto de Fisher como un instrumento de análisis cuantitativo de la BO que, a su vez, permite analizar con más detalle la implicación de los estudiantes con la $\mathrm{BO}$ y sus intentos de resolver un determinado problema. Con ello, y aunque desviándonos de nuestra pregunta inicial, cobra sentido y se hace evidente la posibilidad que en investigaciones de carácter cualitativo puedan incluirse análisis cuantitativos, independientemente del tamaño de la muestra.

A su vez, dicha correspondencia ha permitido confirmar la consideración de la BO por parte de los alumnos, en tanto que las acciones marcadas son observables en las producciones de los alumnos. A nuestro entender, este hecho se convierte en evidencia de la actuación destacada de la $\mathrm{BO}$ en elementos metacognitivos de los alumnos, en tanto que les ha llevado a tomar consciencia de su proceso de resolución y de las 
decisiones tomadas en cada momento, y, por lo tanto, a cuestionarse las acciones desarrolladas. De manera paralela, se percibe como un indicador de la toma de responsabilidad, que, a su vez, implica una progresiva reducción de la guía directa y específica del profesor. Además, la BO ha respondido de manera contingente a las necesidades de los alumnos, en tanto que éstos fueron capaces de conectar las acciones de la base de orientación con su propia actividad. En este sentido, observamos como la BO promueve los características básicas observadas en los andamiajes educativos (van de Pol et al., 2010).

De acuerdo con ello, y en respuesta a los principios de autorregulación (Jorba y Sanmartí, 2004), el estudio realizado nos da indicios de como la $\mathrm{BO}$, a través de la columna de seguimiento, ha conseguido comunicar los objetivos a los alumnos quienes, a su vez, los han podido confrontar con la consecución de los mismos. En cuanto a la apropiación por parte de los alumnos del propio instrumento, y más que ello, del dominio de la anticipación y planificación de las acciones, este estudio apunta a su adquisición por parte de los alumnos, si bien cabría un análisis longitudinal para asegurar la adquisición de las mismas.

Al analizar con más profundidad la implicación con las acciones que conlleva la $\mathrm{BO}$, constatamos cómo la realización de siete o más acciones de las doce que conlleva la $\mathrm{BO}$ se presenta como una condición necesaria, aunque no suficiente, para finalizar la resolución de problema y obtener soluciones satisfactorias a las cuestiones propuestas en él. A su vez se desprende un hecho relevante sobre el papel destacado que toman las acciones de comprobación para un uso efectivo de la base de orientación. Más concretamente, y centrándonos en los alumnos que no obtuvieron soluciones correctas, observamos cómo la importancia de comprobar surge como un indicador del número de acciones de la $\mathrm{BO}$ en las que se involucran los alumnos y la probabilidad de que los alumnos den por finalizada la resolución del problema, aunque sea de forma incorrecta. En este sentido, parece como si dichas acciones proporcionaran cierta autonomía a los alumnos para proseguir con su resolución a pesar de sus dificultades, pero sin garantía de que sea necesariamente de manera efectiva, lo que requiere de un análisis más profundo.

Con ello, parece manifestarse que la autorregulación involucra no solamente el conocimiento y la habilidad metacognitiva, sino que implica cierto sentido de autoeficacia y agencia personal, que se ven influenciados por procesos motivacionales y conductuales (Zimmerman, 1995), y 
en los que aparentemente el uso de la BO también puede influir (VIIlalonga Pons y Andrews, 2017); por consiguiente, estos aspectos no deben descuidarse al generar y analizar el uso de una base de orientación.

Finalmente, es necesario atender el elevado número de intentos fallidos de resolución. Entendemos que tanto el hecho de que el problema estuviese en el límite de la competencia de resolución de problemas de los alumnos, como la constatación de un nivel de compromiso bajo de los alumnos con las dimensiones segunda y cuarta, de las que se desprenden las acciones dedicadas a la comprobación, aparecen como motivos razonables que lo explican. De ello se intuye una vinculación entre el compromiso con dichas dimensiones y la obtención de respuestas correctas a un problema.

Con todo ello, notamos que medir el grado de implicación de los alumnos en el uso de una base de orientación es complejo. Sentimos que no es sencillo obtener pruebas consistentes de la eficacia y solvencia del uso de una base de orientación para la resolución de un problema en el conjunto de los alumnos que integran un grupo. De hecho, no es algo nuevo puesto que estudios anteriores ya han mencionado que el impacto del andamiaje educativo es una tarea compleja de evaluar (van de Pol et al., 2010). Por ello se hace necesaria, no solo la realización de estudios longitudinales que permitan analizar la consideración consecutiva de la BO para la resolución de distintos problemas por parte de los alumnos, sino también otros análisis particulares de la aplicación de las distintas acciones y su posible efecto en las respuestas de los alumnos.

Aunque no podemos dar una respuesta contundente en cuanto a la efectividad de la base de orientación considerada como auto-andamiaje para la resolución de problemas de matemáticas, estos primeros intentos de medir su impacto, nos llevan a poder afirmar que el uso de la BO presentada en este caso concreto, sí ha actuado en este sentido.

Finalmente, observamos cómo las acciones sobre las que está diseñada la base de orientación actúan como indicadores para los docentes con los que analizar la implicación de sus alumnos en la actividad que se pretende y con ello evaluar y readecuar la propia base de orientación. En este sentido, la base de orientación facilita a los docentes parámetros medibles como soporte para tomar decisiones, hacer diagnósticos, y adaptar el propio instrumento. De acuerdo con ello, el uso eficiente de una base de orientación por parte de los docentes se convierte, más allá de un instrumento regulador del aprendizaje de los alumnos, en un ins- 
trumento válido para la educación basada en evidencias (Páramo y Hederich, 2014), con las que el docente puede observar la realidad de su práctica e introducir cambios de acuerdo a las necesidades observadas.

\section{Referencias}

Blanco, A. (2008). Las rúbricas un instrumento útil en la evaluación de competencias. En L. Prieto, L., Blanco, A., Morales, P. (Coords.) La enseñanza universitaria centrada en el aprendizaje: estrategias útiles para el profesorado. Barcelona, España: Octaedro Editorial.

Carrillo, J., y Guevara, F. (1996). Un instrumento para evaluar la resolución de problemas. UNO Revista de Didáctica de las Matemáticas, 8, 65-81.

Coltman, P., Petyaeva, D., y Anghileri, J. (2002). Scaffolding learning through meaningful tasks and adult interaction. Early Years, 22(1), 39-49.

De Corte, E., Verschaffel, L., y Op'tEynde, P. (2000). Self-regulation: A characteristic and a goal of mathematics learning. En M. Boekaerts, P. R. Pintrich y M. Zeidner (Eds.), Handbook of self-regulation (pp. 687-726). San Diego: Academic Press.

De Corte, E., y Verschaffel, L. (2003). El desarrollo de habilidades de autoregulación en la solución de problemas matemáticos. Pensamiento Educativo, 32, 286-305.

Departament d'Ensenyament. Generalitat de Catalunya. (2016). Un currículum competencial per a l'educació primària. Un pas més cap a l'assoliment de les competències bàsiques. Recuperado de http://ateneu.xtec.cat/wikiform/wikiexport/_media/cursos/ curriculum/inf_pri/cape/info_prima_ria_29_6.pdf [enero 2018].

Kirkman, T.W. (1996) Exact $r \times c$ Contingency Table: How many rows? columns? Statistics to Use. Recuperado de http://www.physics.csbsju.edu/stats/exact_NROW_NCOLUMN_form.html [enero 2018].

Jorba, J., y Sanmartí, N. (2004). La función pedagógica de la evaluación. En A. Parcerisa, M. Rovira (Eds.) Evaluación como ayuda al aprendizaje (pp. 21-44). Barcelona, España: GRAÓ

Lester Jr., F.K., y Cai, J. (2016). Can Mathematica IProblem Solving Be Taught? Preliminary Answers from 30 Years of Research. En P. Felmer, E. Pehkonen, y J.Kilpatrick (Eds.), Posing and solving mathematical problems. Advances and New Perspectives (pp. 117-135).Switzerland: Springer

Lester, F. K., y Kroll, D. L. (1991). Evaluation: A new vision. The Mathematics Teacher, 84(4), 276-284.

Llopis, J. (2013). Test exacto de Fisher. La estadística: una orquestra hecha instrumento. Recuperdo de https://estadisticaorquestainstrumento.wordpress.com/2013/01/21/ test-exacto-de-fisher/ [mayo 2017].

Mason, J. (2016a). Part 1 Reaction: ProblemPosing and Solving Today En P. Felmer, E. Pehkonen, y J. Kilpatrick, J. (Eds.), Posing and solving mathematical problems. Advances and New Perspectives (pp. 109-113). Switzerland: Springer

Mason, J. (2016b). When Is a Problem...? "When" Is Actually the Problem! En P. Felmer, 
E. Pehkonen, y J. Kilpatrick, J. (Eds.), Posing and solving mathematical problems. Advances and New Perspectives (pp. 263-285).Switzerland: Springer

Mason, J., Burton, L., y Stacey, K. (1982). Thinking mathematically. Wokingham: Addison-Wesley.

Niss, M., y Højgaard, T. (Eds.) (2011). Competencies and Mathematical Learning. Ideas and inspiration for the development of mathematics teaching and learning in Denmark, IMFUFA tekst 485/2011. Roskilde, Denmark: Department of Science, Systems and Models, Roskilde University.

Panaoura, A. (2016). Self-Regulatory Strategies During Problem-Solving by Using an Inquiry-Based Approach: "Make Sense of Problems and Persevere in Solving Them" . In K. Newton (Ed.), Problem-Solving: strategies, challenges andoutcomes (pp. 197-210). Nova science publishers

Páramo, P., y Hederich. C. (2014). Educación basada en la evidencia. Revista Colombiana de Educación, 66, 13-16.

Pólya, G. (1945). How to solve it. Princeton: Princeton University Press.

Ponte, J. P. (2007). Investigations and explorations in the mathematics classroom. ZDM Mathematics Education, 39, 419-430.

Rosli, R.,Goldsby, D.yCapraro, M.M. (2013). Assessing Students' Mathematical ProblemSolvingand Problem-Posing Skills. Asian Social Science, 9(16),54-60.

Sanmartí, N., y Jorba, J. (1995). Autorregulación de los procesos de aprendizaje y construcción de conocimientos. ALAMBIQUE Didáctica de las Ciencias Experimentales, 4, 59-77.

Sanmartí, N. (2007). Evaluar para aprender. 10 ideas clave. Barcelona, España: Graó.

Sanmartí, N. (2010). Avaluar per aprendre. L'avaluació per millorar els aprenentatges de l'alumnat en el marc del currículum per competències. Recuperado de http://xtec. gencat.cat/web/.content/alfresco/d/d/workspace/SpacesStore/0024/fc53024f-626e423b-877a-932148c56075/avaluar_per_aprendre.pdf [enero 2018].

Schoenfeld, A. H. (1992). Learning to think mathematically: Problem solving, metacognition, and sense-making in mathematics. En D. Grouws (Ed.), Handbook for Research on Mathematics Teaching and Learning (334-370). New York: MacMillan.

Schoenfeld, A. H. (2013). Reflections on problem solving: theory and practice. The Mathematics Enthusiast, 10(1y2), 9-34.

Smit, R., Bachmann, P., Blum, V., Birri, T., y Hess, K. (2017). Effects of a rubric for mathematical reasoning on teaching and learning in primary school. Instructional Science, 45, 603-622.

Stacey, K. (2016). Reaction: Teachers, ProblemPosing and Problem-Solving En P. Felmer, E. Pehkonen, y J. Kilpatrick, J. (Eds.), Posing and solving mathematical problems. Advances and New Perspectives (pp. 387-391). Switzerland: Springer

Van de Pol, J., Volman, M., y Beishuizen, J. (2010). Scaffolding in teacher-student interaction: A decade of research. Educational Psychology Review, 22(3), 271-296.

Villalonga Pons, J. (2017). La competencia matemática. Caracterización de actividades de aprendizaje y de evaluación en la resolución de problemas en la enseñanza obligatória. Tesis doctoral. Universitat Autònoma de Barcelona.

Villalonga Pons, J.,y Andrews, P. (2017). Self-scaffolding students' problem solving: Tes- 
ting an orientation basis. En T. Dooley y G. Gueudet (Eds.), Proceedings of theTenth Congress of the European Society for Research in Mathematics Education. Dublin, Ireland: DCU Institute of Education and ERME.

Villalonga Pons, J. y Andrews, P. (2018). Supporting students' mathematical problem solving: The key role of different forms of checking as part of a self-scaffolding mechanism. En E. Norén, H. Palmér y A. Cooke, Nordic Research in Mathematics Education: Papers of NORMA 17. The Eighth Nordic Conference on Mathematics Education Stockholm, May 30 - June 2, 2017 (pp. 79-88). Estocolmo.

Villalonga Pons, J. y Deulofeu, J. (2015). La base de orientación, una herramienta para ayudar al alumnado a resolver problemas. En FESPM y SEMRM (Eds.) Actas JAEM 2015: 17 Jornadas para el Aprendizaje y la Enseñanza de las Matemáticas (p. 36, 16 páginas). Cartagena, España.

Villalonga Pons, J.,y Deulofeu, J. (2017a). Representar problemas usando una base de orientación. UNO. Revista de Didáctica de las Matemáticas, 75, 59-65.

Villalonga Pons, J., y Deulofeu, J. (2017b). La Base de Orientación en la Resolución de Problemas: "cuando me Bloqueo o me Equivoco". Journal of Research in Mathematics Education, REDIMAT, 6(3), 256-282.

Wood, D., Bruner, J., y Ross, S. (1976). The role of tutoring in problem solving. Journal of Child Psychology and Psychiatry, 17, 89-100.

Zimmerman, B. J. (1995). Self-Regulation Involves More Than Metacognition: A Social Cognitive Perspective. Educational Psychologist,30(4), 217-221. 


\section{Anexo:}

Problemas trabajados durante la implementación.

\section{Problema 1. Multiplicar dos números de dos cifras}

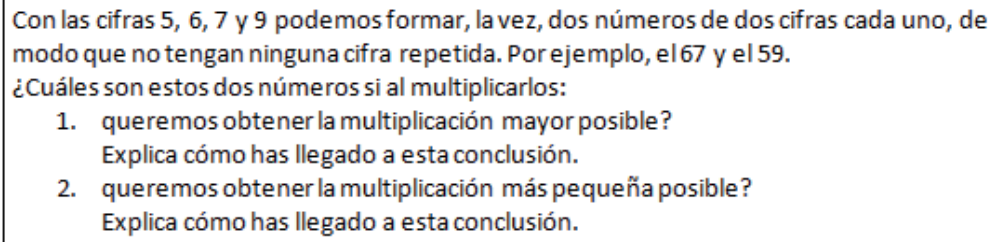

1. queremos obtener la multiplicación mayor posible? Explica cómo has llegado a esta conclusión.

2. queremos obtener la multiplicación más pequeña posible? Explica cómo has llegado a esta conclusión.

Figura 1. Enunciado del $1^{\text {er }}$ problema, traducido al español del original catalán.

\section{Problema 2. El precio del USB}

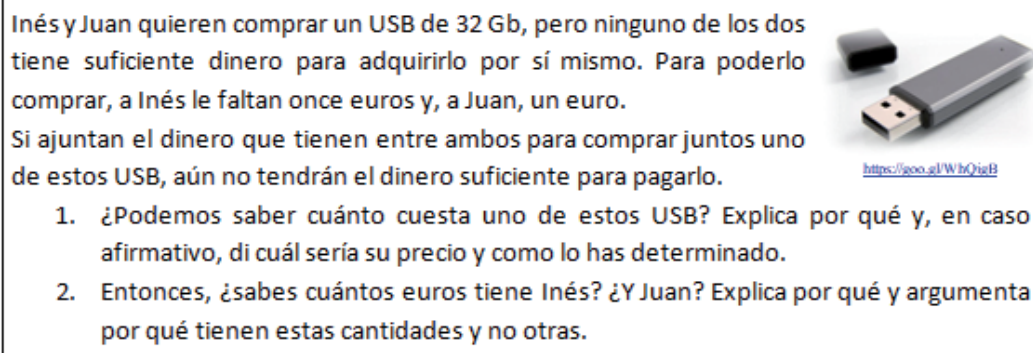

1. ¿Podemos saber cuánto cuesta uno de estos USB? Explica por qué y, en caso afirmativo, di cuál sería su precio y como lo has determinado.

2. Entonces, ¿̇sabes cuántos euros tiene Inés? ¿Y Juan? Explica por qué y argumenta por qué tienen estas cantidades y no otras.

Figura 2. Enunciado del $2^{\circ}$ problema, traducido al español del original catalán.

\section{Problema 3. La manta de soles y lunas}

Arnau ha comprado una alfombra muy grande de $6 \mathrm{~m}$ de largo y de 3,6 $\mathrm{m}$ de ancho. La alfombra, tal como se puede ver con la parte desplegada que muestra la imagen, está formada de pequeños cuadrados que contienen el dibujo de un Sol o de una Luna.

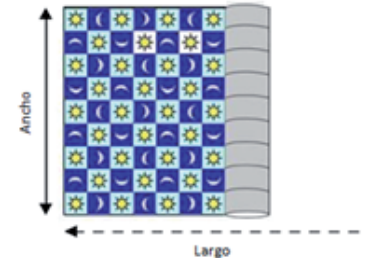

1. Cuando la alfombra esté desplegada del todo, ¿̇cuántos cuadraditos habrá en total? Explica cómo lo has hecho para saberlo.

2. De todos estos cuadraditos, ¿̇e puede saber cuántos contendrán un Sol y cuántos una Luna? Razona por qué sí o por qué no y, en caso de que se pueda, explica qué

Figura 3. Enunciado del $3^{\text {er }}$ problema, traducido al español del original catalán. 
